\title{
CERAMBYCIDAE (COLEOPTERA) DA COLÔMBIA. II. IBIDIONINI (CERAMBYCINAE)
}

\author{
Ubirajara R. Martins $\mathbf{s}^{1,3}$ \\ Maria Helena M. Galileo ${ }^{2,3}$
}

\begin{abstract}
CERAMBYCIDAE (COLEOPTERA) OF COLOMBIA. II. IBIDIONINI (CERAMBYCINAE). Colombian records are given for Hexoplon albipenne Bates, 1872; H. venus Thomson, 1864; Gnomidolon rubricolor Bates, 1870; Tropidion brunneiceps (Thomson, 1865); Thoracibidion buquetii (Thomson, 1867); T. io (Thomson, 1867); Rhysium bivulneratum (Thomson, 1867); $R$. guttiferum (Thomson, 1867); Compsibidion maronicum (Thomson, 1867); C. varipenne Martins, 1969; ?Compsa flavofasciata (Thomson, 1867); Neocompsa squalida (Thomson, 1867); Heterachthes signaticollis (Thomson, 1865). New species described: Gnomidolon bellus, from Tolima and Neocompsa longipilis, from Santander.
\end{abstract}

KEYWORDS. Cerambycidae, Colombia, Ibidionini, new records, new species.

\section{INTRODUÇÃO}

A primeira contribuição sobre novos registros e novas espécies para os cerambicídeos da Colômbia tratou da tribo Eburiini (Martins \& Galileo, 2002) com acréscimos de novos dados e novos táxons ao levantamento da fauna cerambicidológica colombiana (MARTínEZ, 2000).

Dando continuidade ao estudo do material enviado por Claudia Martínez e pertencente ao Instituto de Investigaciones de Recursos Biológicos "Alexander von Humboldt", Villa de Leyva (IAHC), Facultad de Agronomia, Universidad Nacional de Colombia, Santafé de Bogotá (UNAB) e à coleção Gilberto Mendoza, Santafé de Bogotá (GMIC), estudaremos a tribo Ibidionini (Cerambycinae). Parte desse material foi retido no Museu de Zoologia, Universidade de São Paulo (MZSP) e Museu de Ciências Naturais, Fundação Zoobotânica do Rio Grande do Sul (MCNZ).

A menção das províncias da Colômbia segue a ordem adotada por Zanella et al. (2000). Outra sigla mencionada no texto (BMNH) corresponde ao The Natural History Museum, Londres.

1. Museu de Zoologia, Universidade de São Paulo, Caixa Postal 42594, São Paulo, SP, 04299-970, Brasil.

2. Museu de Ciências Naturais, Fundação Zoobotânica do Rio Grande do Sul, Caixa Postal 1188, Porto Alegre, RS, 90001-970, Brasil.

3. Pesquisador CNPq. 


\section{Divisão}

\section{Hexoplon albipenne Bates, 1872}

Hexoplon albipenne Bates, 1872:179; Joly, 1991:12; Monné, 1993:6 (cat.); Martínez, $2000: 88$ (distr.). Hexoplon colombianum Martins, 1959:340; MARTins, 1967:99, figs. 55, 60-63 (sin.).

Espécie muito variável no padrão de colorido dos élitros (MARTINs, 1967) e largamente distribuída na América Central (Guatemala ao Panamá), na Colômbia e na Venezuela. Joly (1991), além de discutir a variabilidade do colorido, mencionou-a para Venezuela nos estados de Zulia, Falcón, Lara, Carabobo e Aragua. As localidades já mencionadas para a Colômbia situam-se em Magdalena (Aracataca) e Cundinamarca (Fusagasugá). Acrescenta-se uma nova localidade para Magdalena para espécime cujo padrão de colorido elitral é igual ao mostrado por MARTiNs (1967, fig. 62), baseado em exemplar de Cundinamarca.

Material examinado. COLÔMBIA, Magdalena: PNN (Parque Nacional Natural) Tayrona (Pueblito, $1^{\circ} 20^{\prime} \mathrm{N}, 7^{\circ} 02^{\prime} \mathrm{W}, 225 \mathrm{~m}$ ), O', 31.I.-21.II.2001, R. Henriquez col., “Malaise” (IAHC).

\section{Hexoplon venus Thomson, 1864}

Hexoplon venus Thomson, 1864:219; Martins, 1967:131, fig.82, est.3 fig.2; Joly, 1991:13; MonnÉ, 1993:10 (cat.); MartínEZ, 2000:88 (distr.).

Gnomidolon venus; LACORDAIRE, 1869:330.

Originalmente descrita da Guiana Francesa e mencionada por MARTins (1967) para a Cundinamarca (Santafé de Bogotá). MARTínez (2000) mencionou-a para a Colômbia sem precisar localidades. JoLY (1991) registrou-a para a Venezuela (Aragua).

Material examinado. COLÔMBIA, Cundinamarca: La Meza (Laguna Pedro Palo, 04³8'05"N, $\left.74^{\circ} 27^{\prime} 57^{\prime \prime} \mathrm{W}, 1298 \mathrm{~m}\right)$, , , 16.IV.1996, I. Ospina col. (UNAB); Fusagasugá $\left(04^{\circ} 20^{\circ} 49^{\prime \prime} \mathrm{N}, 74^{\circ} 21^{\prime} 53^{\prime \prime} \mathrm{W}\right.$, $1731 \mathrm{~m}$ ),, , 2.X.1993, P. Osorio col. (MZSP).

\section{Gnomidolon rubricolor Bates, 1870}

Gnomidolon rubricolor Bates, 1870:286; Martins, 1967:206; Joly, 1991:20; MonNÉ, 1993:16 (cat.). Gnomidolon pygmaeum Martins, 1962:275, fig.7; Martins, 1967:206 (sin.).

Esta espécie originalmente descrita do Brasil (Pará, Tapajós) foi assinalada, posteriormente, por MARTins (1967) para a Venezuela (Amazonas, "Alto Cunucunuma"), Peru (Loreto e San Martin) e Brasil (Amazonas). JoLY (1991) ampliou a distribuição na Venezuela, arrolando-a para Amazonas, "Cerro de La Neblina”. É registrada, pela primeira vez, para a Colômbia.

Material examinado. COLÔMBIA, Amazonas: PNN (Parque Nacional Natural) Amacayacu Matamata $\left(03^{\circ} 23^{\prime} \mathrm{S}, 70^{\circ} 06^{\prime} \mathrm{W}, 150 \mathrm{~m}\right), \sigma^{\circ}, 27 . \mathrm{III}$ a 03.IV.2000, A. Parente col., armadilha Malaise (IAHC).

Iheringia, Sér. Zool., Porto Alegre, 92(4):11-18, 30 de dezembro 2002 


\title{
Gnomidolon bellus sp. nov.
}

\author{
(Fig. 1)
}

Cabeça avermelhada. Antenas amareladas ou alaranjadas com escapo habitualmente mais escuro. Protórax avermelhado. Pronoto sem pubescência, muito liso e brilhante, prosterno com pubescência apenas no processo prosternal. Élitros (fig. 1) avermelhados no quinto basal; em cada élitro, uma faixa preta na sutura, desde o quarto anterior até o meio, onde se volta descendente da sutura para a margem (em conjunto com a correspondente do outro élitro, forma um " $X$ "); mancha branca lateral antes do meio (envolvida pelos braços do "X" preto); uma faixa branco-amarelada, oblíqua, ascendente da margem para a sutura imediatamente atrás da borda posterior da faixa preta; terço apical avermelhado, exceto na ponta, que é ocupada por área branco-amarelada. Dorso de cada élitro, no nível do meio, com duas fileiras longitudinais de pontos pilíferos. Extremidades elitrais obliquamente truncadas com espinho no lado externo. Mesosterno, metasterno e abdômen avermelhados. Pernas amareladas; fêmures posteriores pretos com o terço basal amarelado. Mesofêmures com curto espinho apical interno. Metafêmures com dois espinhos: o externo com o dobro do comprimento do interno.

Dimensões, em mm, ơ. Comprimento total, 9,0-8,2; protórax: comprimento 2,3-2,1; maior largura, 1,2-1,1; comprimento do élitro, 5,6-5,3, largura umeral, 1,8-1,5.

Material-tipo. COLÔMBIA, Tolima: Armero (Guayabal Mendez, Hacienda Bremen, 0505'17"N, $74^{\circ} 45^{\prime} 12^{\prime}$ W, $300 \mathrm{~m}$ ), holótipo $0^{\circ}$, 17.IX.1997, F. Fernández col., armadilha Malaise (IAHC); parátipo ○', mesmos dados do holótipo (MZSP).

Discussão. Gnomidolon bellus assemelha-se a G. rubricolor Bates, 1870 pelo número de fileiras longitudinais de pêlos nos élitros e pelo padrão do colorido elitral. Difere pela cor das pernas: as anteriores e as médias, amareladas e os metafềmures, pretos em grande extensão; pelos élitros com a faixa (em "X") preta mais longa (fig. 1) e mancha esbranquiçada láteroanterior bem mais alongada. Em G. rubricolor todas as pernas são avermelhadas e o " $\mathrm{X}$ " preto dos élitros envolve uma área branca lateral menor.

\section{III ${ }^{\mathrm{a}}$ Divisão}

\section{Tropidion brunniceps (Thomson, 1865)}

Ibidion brunniceps THомsом, 1865:571.

Ibidion (Tropidion) brunniceps; THомsоN, 1867:138.

Ibidion (Compsidion) brunniceps; LACORDAIRE, 1869:332.

Tropidion brunniceps; Martins, 1968:407, figs. 228, 229; Joly, 1991:29; Monné, 1993:22 (cat.); Martínez, 2000:89 (distr.).

Originalmente descrita de Bogotá e Cundinamarca, Colômbia. MarTins (1968) arrolou-a para o Panamá e colocou em dúvida a procedência "Brasil" baseada em material do BMNH. Joly (1991) registrou para a Colômbia (Huila) e Venezuela (Zulia). MARTínEZ (2000) assinalou-a para Atlántico, Colômbia.

Material examinado. COLÔMBIA, Risaralda: Pereira (Dos Quebradas, 04ํำ $49^{\prime} 02^{\prime \prime} \mathrm{N}$, 7541'44"W, $1420 \mathrm{~m}$ ), O', 27.XII.1997, H. Ocampo col. (UNAB); Boyaca: Ráquira (05³2'26"N, $\left.73^{\circ} 38^{\prime} 11^{\prime \prime W}, 2152 \mathrm{~m}\right)$,, , 17.IV.1992, A. Jiménez col. (MZSP); Tolima: Honda $\left(05^{\circ} 12^{\prime} 25^{\prime \prime} \mathrm{N}\right.$, 7444'28"W, 225 m), P, 24.VI.1969, L. Rico \& C. Cujia col. (MZSP); departamento ?: San Cayetano, ९, 7.VI.1971, J. Rodriguez col (UNAB). 


\section{Thoracibidion buquetii (Thomson, 1867)}

Ibidion (Tropidion) Buquetii THOMson, 1867:139.

Ibidion griseicolle BATES, 1872:180; MARTINS, 1960b:107 (sin.).

Ibidion Buquetii; Tномson, 1878:6.

Octoplon Buqueti; Gounelle, 1909:664.

Thoracibidion buqueti; MARTINS, 1960b:107, fig.1; Joly, 1991:40; MonNÉ, 1993:31 (cat.).

Martins (1968) citou T. buquetii para o México, Nicarágua, Costa Rica, Panamá, Colômbia (Magdalena, Santander, Caldas e Cundinamarca), Equador e Guiana Francesa. MarTins (1971b) acrescentou a ocorrência no Peru (Lima). Joly (1991) assinalou-a para a Venezuela (Zulia) e para o Equador (Los Ríos). MonNÉ (1993) e MARTínEZ (2000) repetiram estas procedências.

Material examinado. COLÔMBIA, Santander: Barbosa (055' $\left.58^{\prime} \mathrm{N}, 7^{\circ} 37^{\prime} 16^{\prime \prime} \mathrm{W}, 1588 \mathrm{~m}\right)$, , 5.XI.1998, L. Banda col. (MZSP); Cundinamarca: Sasaima (0457'59" N, 76²6’15" W, $1221 \mathrm{~m})$, §, 18.I.1976, J. Duarte col. (UNAB).

\section{Thoracibidion io (Thomson, 1867)}

Ibidion (Tropidion) Io ThOMSON, 1867:141.

Ibidion Jo; GEMMINGER \& HAROLD, 1872:2829 (cat.).

Octoplon rugicolle BATES, 1870: 296; MARTINS, 1967:537 (sin.).

Ibidion Io; THOMSON, 1878:6.

Thoracibidion io; Martins, 1960b:106, fig. 6; Joly, 1991:37; MonNÉ, 1993:32 (cat.); MartínEZ, $2000: 89$ (distr.).

Espécie largamente distribuída na Colômbia, Venezuela, Guiana, Suriname, Guiana Francesa, Peru, Brasil (do Amazonas ao Rio de Janeiro). Joly (1991) apresentou novas ocorrências para a Venezuela (Zulia, Lara, Portuguesa, Aragua, Táchira, Miranda, Guárico, Barinas) e MARTínez (2000) acrescentou Meta na Colômbia.

Material examinado. COLÔMBIA, Cundinamarca: La Mesa $\left(04^{\circ} 38^{\prime} 05^{\prime \prime} \mathrm{N}, 7^{\circ} 27^{\prime} 57^{\prime \prime} \mathrm{W}, 1298\right.$ m), ơ, 17.VII.1976, L. Rodríguez col. (MZSP); Tolima: Espinal $\left(04^{\circ} 09^{\prime} 10^{\prime \prime N}, 74^{\circ} 53^{\prime} 19^{\prime \prime} \mathrm{W}\right.$, , 30.III.1971, H. Alarcón col. (UNAB).

\section{Divisão}

\section{Rhysium bivulneratum (Thomson, 1867)}

Ibidion (Tropidion) bivulneratum THOMSON, 1867:141.

Ibidion inarmatum Martins, 1960a:94, fig. 3; Martins, 1969:655 (sin.).

Rhyzium bivulneratum; MARTINs, 1969:655, fig. 349.

Rhysium bivulneratum; MonNé, 1993:39 (cat.); MARTínez, 2000:90 (distr.).

Rhysium bivulneratum foi descrita originalmente fundamentada num exemplar do "Brésil" e citada para o Brasil por MARTINS (1969) com base em três exemplares que não possuíam outros dados de procedência, portanto acreditamos que a espécie não ocorra nesse país, sendo conhecida apenas da Colômbia (Antioquia, Cundinamarca e Tolima). MARTínez (2000) citou-a para Cundinamarca.

Material examinado. COLÔMBIA, Cundinamarca: Fusagasugá $\left(04^{\circ} 20^{\prime} 49^{\prime \prime} \mathrm{N}, 7^{\circ} 21^{\prime} 53^{\prime \prime} \mathrm{W}\right.$, 1731 m), O', 03.VI.1994, D. Aponte col. (MZSP); Y, 12.IV.1992, M. Chaves col. (MZSP); Y, 13.X.1977, Trujillo col. ("tallo hierba, día seco") (UNAB); Silvania (04²4’21"N, 74²3’24"W, $1470 \mathrm{~m}), \sigma^{\circ}$, 02.VI.1977, F. Daza \& R. García col. (UNAB).

Iheringia, Sér. Zool., Porto Alegre, 92(4):11-18, 30 de dezembro 2002 

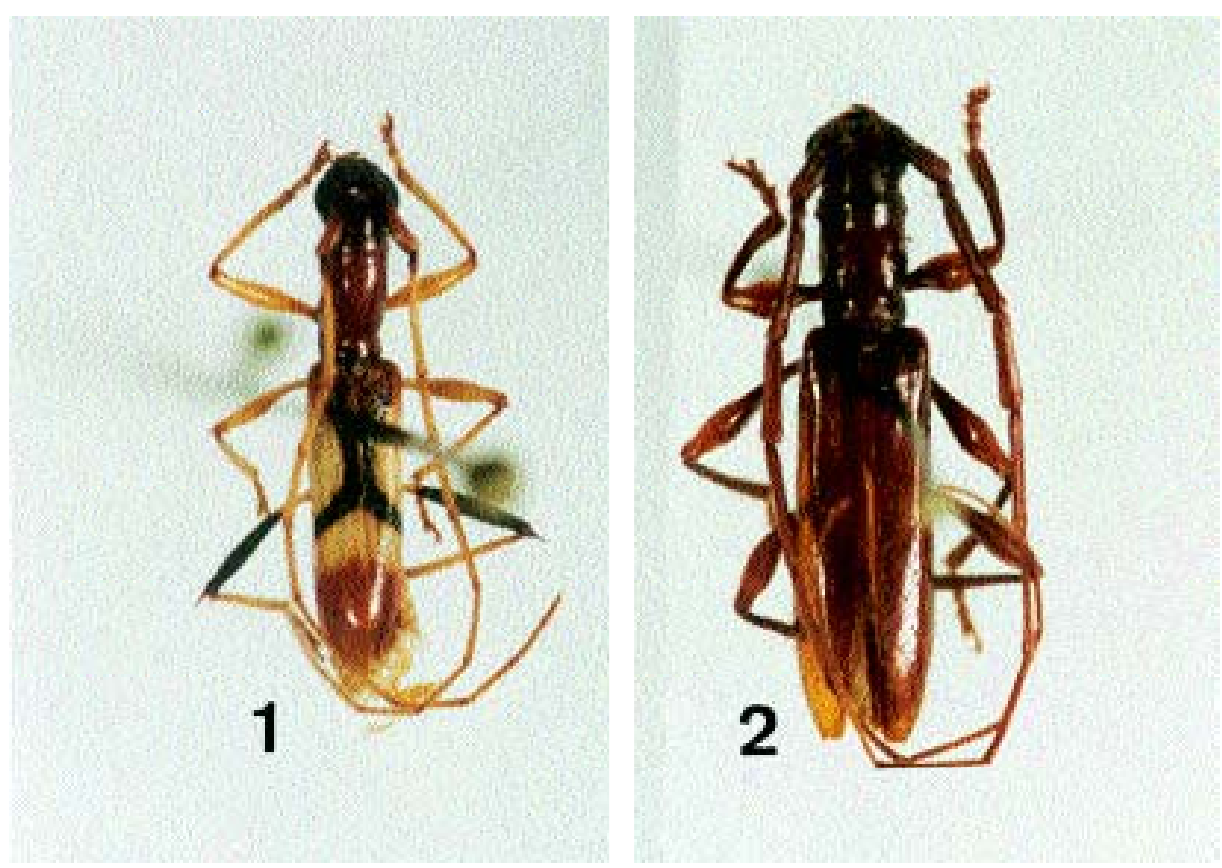

Figs.1-2. 1, Gnomidolon bellus sp. nov., holótipo on, comprimento $9 \mathrm{~mm}$; 2, Neocompsa longipilis sp. nov., holótipo ó, comprimento $11,8 \mathrm{~mm}$.

\section{Rhysium guttiferum (Thomson, 1867)}

Ibidion (Tropidion) guttiferum THомsоN, 1867:142.

Rhyzium guttiferum; Martins, 1969:658, figs. 349, 353.

Rhysium guttiferum; MonNÉ, 1993:39 (cat.); MartíneZ, 2000:89 (distr.).

Conhecida somente da Colômbia: Cundinamarca e Boyacá; amplia-se sua distribuição para Huila.

Material examinado. COLÔMBIA, Cundinamarca: Santafé de Bogotá (Barrio Lisboa), 0', 1993, Corradine M. col. (GMIC); O', 28.VII.1989, Corradine M. col. (GMIC); (perímetro urbano), P, IV.1987, G. Alrobre ? col. (GMIC); Boyacá: San José de Pare $\left(06^{\circ} 01^{\prime} 12^{\prime \prime N}\right.$, $\left.73^{\circ} 33^{\prime} 03^{\prime \prime} \mathrm{W}, 1545 \mathrm{~m}\right), 0^{\circ}, 7 . V I .1972$, C. Muñoz col. (UNAB); Huila: Neiva $\left(02^{\circ} 55^{\prime} 46^{\prime \prime N}\right.$, $75^{\circ} 17^{\prime} 31^{\prime \prime} \mathrm{W}, 442 \mathrm{~m}$ ),, , 6.VI.1974, [sem nome de coletor] (MZSP).

\section{Compsibidion maronicum (Thomson, 1867)}

Ibidion maronicum THOмson, 1867:135.

Comsibidion maronicum; Martins, 1969:783; Joly, 1991:47; MonNÉ, 1993:44 (cat.).

Ibidion Herse ThOMSON, 1867:136; MARTins, 1969:783 (sin.).

Ibidion oedicneme BATES, 1870:298; MARTINS, 1964:207 ( $\sin$. ).

Ibidion rubellum BATES, 1870:298; MARTINS, 1964:207 (sin.).

Ibidion leprieuri BATES, 1870:299; MARTINS, 1964:207 (sin.).

Ibidion dilectum BATES, 1870:299; MARTINs, 1964:207 (sin.).

Heterachtes deliciolus BATES, 1870:305; Martins, 1969:783 (sin.). 
Ocorrente principalmente na Amazônia, foi registrada por MARTINS (1969) para Trinidad y Tobago, Venezuela, Suriname, Guiana Francesa, Peru, Brasil (Amazônia) e Bolívia . Registra-se agora, pela primeira vez, para a Colômbia.

Material examinado. COLÔMBIA, Vichada: PNN (Parque Nacional Natural) Tuparro (Cerro Tomás, $05^{\circ} 21^{\prime} \mathrm{N}, 67^{\circ} 51^{\prime} \mathrm{W}, 140 \mathrm{~m}$ ), , , 29.XI-08.XII.2000, W. Villalba col., armadilha Malaise (IAHC); Amazonas: PNN Amacayacu Matamata $\left(03^{\circ} 23^{\prime} \mathrm{S}, 70^{\circ} 06^{\prime} \mathrm{W}, 150 \mathrm{~m}\right), 3$ 9, 27.III a 03.IV.2000, A. Parente col., armadilha Malaise (19 IAHC; 2 MZSP).

\section{Compsibidion varipenne Martins, 1969}

Compsibidion varipenne Martins, 1969:742, figs. 395, 396, 403; Monné, 1993:48 (cat.).

Originalmente descrita de Maracay (El Limón, 450 m), Aragua, Venezuela. Registrase, pela primeira vez, para a Colômbia.

Material examinado. COLÔMBIA, Magdalena: PNN Tayrona (Pueblito, $11^{\circ} 20^{\prime} \mathrm{N}, 74^{\circ} 02^{\prime} \mathrm{W}$, 225 m), ९, 31.I.-21.II.2001, R. Henriquez col., armadilha Malaise (IAHC).

\section{Divisão}

\section{? Compsa flavofasciata (Thomson, 1867)}

Ibidion (Compsibidion) flavo-fasciatum Tномson, 1867:152.

Ibidion flavofasciatum; Tномson, 1878:6.

Compsa flavofasciata; Aurivillius, 1912:109 (cat.); Joly, 1991:61, fig.92; MonnÉ, 1993:61 (cat.).

? Compsa flavofasciata; Martins, 1970a:1034, fig.530.

Conhecida da Colômbia (Cundinamarca e Caldas) e da Venezuela (Trujillo) (MarTins, 1970a). Joly (1991) mencionou-a para os estados venezuelanos de Mérida, Trujillo e os colombianos Nariño e Norte de Santander. MArTínez (2000) citou para a Colômbia, Antioquia, Cundinamarca e Tolima.

Material examinado. COLÔMBIA, Cundinamarca: Quipile (Vda. Berlín), Y, 01.XI.2000, G. Manrique \& M. Orozco col. "en cultivo de mora" (UNAB).

\section{Neocompsa squalida (Thomson, 1867)}

Ibidion (Compsibidion) squalidum THомsоN, 1867:151.

Ibidion squalidum; THOMSON, 1878:6.

Compsa squalida; Auriviluius, 1912:110 (cat.).

Neocompsa squalida; Martins, 1965:98; Monné, 1993:66 (cat.); Monné, 2001:23 (hosp.).

Martins (1970a) citou-a para o México, Guatemala, Nicarágua, Costa Rica, Panamá, Colômbia e Venezuela. MARTins (1971b) arrolou-a para El Salvador. Joly (1991) detalhou a distribuição na Venezuela citando Táchira, Falcón, Yaracuy, Aragua e Miranda. MARTínez (2000) registrou-a para a Colômbia (Bolívar).

Material examinado. COLÔMBIA, Cundinamarca: San Joaquin (Vda. La Veja) ơ, 07.VI.1996, E. Gordillo col. (UNAB); Tocaima $\left(04^{\circ} 27^{\prime} 40^{\prime \prime} \mathrm{N}, 74^{\circ} 38^{\prime} 16^{\prime \prime} \mathrm{W}, 400 \mathrm{~m}\right), 0^{\circ}, 17 . X .1994$, C. Forero col. (IHAC); Tolima: Honda $\left(5^{\circ} 12^{\prime} 25^{\prime \prime} \mathrm{N}, 74^{\circ} 44^{\prime} 28^{\prime \prime} \mathrm{W}, 225 \mathrm{~m}\right)$, , 24.VI.1969, L. Rico \& C. Cujía col. (MZSP); Huilla: Garzón $\left(02^{\circ} 12^{\prime} 63^{\prime \prime N} 65^{\circ} 38^{\prime} 25^{\prime \prime W}, 828 \mathrm{~m}\right)$, Y, 04.IV.1969, H. Gómez col. "en pepino" (UNAB).

Iheringia, Sér. Zool., Porto Alegre, 92(4):11-18, 30 de dezembro 2002 
Neocompsa longipilis sp. nov.

(Fig. 2)

Macho. Tegumento avermelhado, unicolor. Cabeça com pontuação grossa, esparsa algo confluente na fronte. Lobos oculares superiores com três fileiras de omatídios. Tubérculos anteníferos agudos. Antenas ultrapassam a extremidade elitral a partir do meio do antenômero VII. Escapo grosseiramente pontuado com sulco raso na metade basal. Antenômeros III a VI engrossados, carenados, com pêlos longos e esparsos. Pronoto com tegumento liso, brilhante, com longos pêlos esparsos e escassa pubescência serícea junto à base. Lados do protórax brilhantes. Prosterno com duas faixas largas, longitudinais de pubescência serícea à frente das procoxas até o meio. Escutelo revestido por pubescência esbranquiçada. Élitros (fig. 2) lisos, brilhantes, pontos restritos aos pilíferos que, no meio dorso de cada élitro, organizamse em duas fileiras longitudinais; pêlos muito alongados e esbranquiçados; extremidades levemente emarginadas com projeção mínima no ângulo externo. Pro- e mesofêmures sem carena anteapical. Metasterno e abdômen esparsamente pubescentes.

Dimensões, em mm, o. Comprimento total, 11,8; protórax: comprimento, 2,5; maior largura, 1,8; comprimento dos élitros, 8,2; largura umeral, 2,5.

Material-tipo. COLÔMBIA, Santander: Macaravita (Hacienda El Cairo), holótipo ơ, XI.1993, D. Corradine col. (GMIC).

Discussão. As espécies de Neocompsa com élitros unicolores e glabros são: $N$. fulgens (Fisher, 1932), N. limatula Martins \& Napp, 1986 e forma unicolor de N. exclamationis (Thomson, 1860). O colorido geral avermelhado de $N$. longipilis permite separá-la imediatamente de $N$. fulgens, ocorrente em Dominica (cabeça e protórax alaranjados e élitros amarelados com estreita orla sutural e margens acastanhadas) e dos unicolores de $N$. exclamationis, registrada para os Estados Unidos e México (colorido geral acastanhado).

Neocompsa longipilis distingue-se de $N$. limatula pela cabeça pontuada; pelos antenômeros III e IV engrossados ( $\widetilde{\sigma}^{\prime}$ ); élitros unicolores com duas fileiras longitudinais de pontos pilíferos, onde se implantam pêlos muito longos e pelas pernas avermelhadas. Em N. limatula, descrita do Brasil (Rondônia e Mato Grosso), a cabeça é muito esparsamente pontuada, os antenômeros III e IV (ơ) são indistintamente engrossados; os élitros são acastanhados, escurecidos nos ápices, cada um com cinco fileiras longitudinais de pêlos curtos e as pernas são acastanhadas.

\section{Heterachthes signaticollis (Thomson, 1865)}

Ibidion signaticolle TномsоN, 1865:572.

Ibidion (Heterachthton) signaticolle; THомson, 1867:148.

Compsa signaticolle; LACORDAIRE, 1869:333.

Heterachtes signaticollis; AurIviluIus, 1912:111 (cat.).

Heterachthes signaticollis; Martins, 1965:213; Joly, 1991:74; MonnÉ, 1993:78 (cat.).

Citada por MARTINs (1970b) para a Venezuela (Mérida e Distrito Federal); em (1971a), arrolou-a para a Colômbia sem detalhar as localidades e em (1971b) citou-a para Venezuela, Miranda. Joly (1991) registrou-a para Mérida, Aragua, Distrito Federal e Miranda, na Venezuela. É feita a primeira citação para a Colômbia com localidade precisa.

Material examinado. COLÔMBIA, Casanare: Yopal (Via la Chaparrera, $05^{\circ} 21^{\prime} \mathrm{N}, 72^{\circ} 24^{\prime} \mathrm{W}$, 350 m), P, 02.V.1998, M. Rodríguez col. (UNAB).

Agradecimentos. A Antonio Santos Silva (MZSP) pela execução das fotografias. O material pertencente ao IHAC foi colecionado com apoio do NSF, DEB 9972024 a Michael Sharkey e Brian Brown.

Iheringia, Sér. Zool., Porto Alegre, 92(4):11-18, 30 de dezembro de 2002 


\section{REFERÊNCIAS BIBLIOGRÁFICAS}

Aurivillius, C. 1912. Coleopterorum Catalogus, pars 39, Cerambycinae. Berlin, W. Junk, 574p.

Bates, H. W. 1870. Contributions to an insect fauna of the Amazon Valley. Trans. ent. Soc. London, London, 1870:243-335.

- 1872. On the longicorn Coleoptera of Chontales, Nicaragua. Trans. ent. Soc. London, London, 1872:163-238.

Gemminger, M. \& Harold, E. von. 1872. Catalogus Coleopterorum... Monachii, Gummi. v. 9, p. 2669-2988.

Gounelle, E. 1909. Liste de cérambycides de la région de Jatahy, État de Goiaz, Brésil. Annls Soc. ent. Fr. Paris, 77:587-688.

Joly, L. J. 1991. Sinopsis de la tribu Ibidionini (Coleoptera, Cerambycidae) en Venezuela. Acta Terramaris, Caracas, (4):1-84.

Lacordaire, J. T. 1869. Genera de Coléoptères... Paris, Roret. v. 8, 552p.

Martínez, C. 2000. Escarabajos longicórnios (Coleoptera: Cerambycidae) de Colômbia. Biota Colombiana, Bogotá, 1(1):76-105.

Martins, U. R. 1959. Ibidionini (Coleoptera, Cerambycinae). IV. Cometários, redescrição e espécies novas do gênero Hexoplon Thomson. Papéis Avuls Zool., São Paulo, 13:339-349.

- 1960a. Ibidionini (Coleoptera, Cerambycinae). X. Novas espécies do gênero Ibidion. Papéis Avuls Zool., São Paulo, 14:93-98.

- 1960b. Ibidionini (Coleoptera, Cerambycinae). XI. Gêneros de pronoto enrugado. Papéis Avuls Zool., São Paulo, 14:105-113.

- 1962. Ibidionini (Coleoptera, Cerambycinae). XV. Novas espécies, notas sinonímicas, redescrições. Papéis Avuls Zool., São Paulo, 14:267-310.

- 1964. Ibidionini (Coleoptera, Cerambycinae). XXIII. Sôbre Ibidion maronicum Thomson, 1867, espécie polimórfica. Papéis Avuls Zool., São Paulo, 16:207-212.

- 1965. Ibidionini (Coleoptera, Cerambycinae). XXIV. Divisão do gênero Compsa Perty, 1832. Papéis Avuls Zool., São Paulo, 17:89-107.

-. 1967. Monografia da tribo Ibidionini (Coleoptera, Cerambycinae). Parte I. Arq. Zool. Est. S. Paulo, São Paulo, 16:1-320.

-. 1968. Monografia da tribo Ibidionini (Coleoptera, Cerambycinae). Parte II. Arq. Zool. Est. S. Paulo, São Paulo, 16:321-630.

- 1969. Monografia da tribo Ibidionini (Coleoptera, Cerambycinae). Parte III. Arq. Zool. Est. S. Paulo, São Paulo, 16:631-877.

- 1970a. Monografia da tribo Ibidionini (Coleoptera, Cerambycinae). Parte IV. Arq. Zool. Est. S. Paulo, São Paulo, 16:879-1149.

- 1970b. Monografia da tribo Ibidionini (Coleoptera, Cerambycinae). Parte V. Arq. Zool. Est. S. Paulo, São Paulo, 16:1151-1342.

-. 1971a. Monografia da tribo Ibidionini (Coleoptera, Cerambycinae). Parte VI. Arq. Zool. Est. S. Paulo, São Paulo, 16:1343-1508.

- 1971b. Notas sobre Cerambycidae VI (Coleoptera, Cerambycidae). Subsídios ao conhecimento dos Ibidionini. Arq. Zool., São Paulo, 21:121-178.

Martins, U. R. \& Galleo, M. H. M. 2002. Cerambycidae (Coleoptera) da Colômbia. I. Eburiini. Iheringia, Sér. Zool., Porto Alegre, 92(4): 5-10.

Monné, M. A. 1993. Catalogue of the Cerambycidae (Coleoptera) of the western hemisphere. Part V. São Paulo, Sociedade Brasileira de Entomologia. 100p.

- 2001. Catalogue of the neotropical Cerambycidae (Coleoptera) with known host plant. Part II: subfamily Cerambycinae, tribes Graciliini to Trachyderini. Publções avul. Mus. nac., Rio de Janeiro, (90):3-119.

Thomson, J. 1864. Systema cerambycidarum. Mém. Soc. r. Sci. Liège, Liège, 19:1-540.

- 1865. Diagnoses d'espèces nouvelles qui séront décrites dans l'appendix du systema cerambycidarum. Mém. Soc. r. Sci. Liège, Liège, 19:541-578.

- 1867. Ibidionitarum species novae. Physis Rec. Hist. Nat., Paris, 1:133-163.

- 1878. Typi cerambycidarum Musei Thomsoniani. Paris, E. Deyrolle. 21p.

Zanella, F. C. V.; Oliveira, M. L. DE \& Gaglione, M. C. 2000. Standizarning lists of locality data for specimens in systematics and biogeography studies of new world taxa. Biogeographica, Paris, 76:145-160.

Recebido em 22.04.2002; aceito em 05.09.2002.

Iheringia, Sér. Zool., Porto Alegre, 92(4):11-18, 30 de dezembro 2002 